\title{
Intervenções Percutâneas na Isquemia Arterial Mesentérica: Indicações, Técnica e Tratamento
}

\author{
Marcos Antonio Marino', Walter Rabelo', Roberto Luiz Marino', Mauro Isolani Pena', \\ Alexandre Von Sperling de Vasconcelos ${ }^{1}$, Roberto José de Queiroz Crepaldi', \\ Guilherme Abreu Nascimento', Ronald de Souza'
}

\section{RESUMO}

Os relatos científicos a respeito da isquemia arterial mesentérica sugerem que essa doença é pouco entendida, muito pouco diagnosticada e raramente tratada, apesar de sua alta letalidade. O prognóstico da isquemia mesentérica dependerá do rápido restabelecimento do fluxo sanguíneo, seja por meio de medidas clínicas, cirúrgicas, endovasculares ou da combinação destas. Neste artigo revisam-se as principais evidências diagnósticas da isquemia mesentérica bem como as opções terapêuticas disponíveis, incluindo a intervenção percutânea como método alternativo à cirurgia, que é associada a significativas taxas de morbidade e mortalidade.

DESCRITORES: Artérias mesentéricas/cirurgia. Artérias mesentéricas/diagnóstico. Isquemia/diagnóstico. Isquemia/cirurgia. Angioplastia com balão. Mesentério/irrigação sanguínea. Fibrinolíticos/uso terapêutico. Tirofiban/administração \& dosagem. Anticoagulantes/uso terapêutico.

"A melhor parte da história da isquemia mesentérica ainda está por ser escrita." Boley, 1997

A pesar dos avanços acerca do diagnóstico por imagem, do tratamento farmacológico e das técnicas de revascularização nas últimas décadas, a insuficiência vascular mesentérica permanece uma condição de alta letalidade. Se por uma lado a isquemia mesentérica é uma afecção rara, correspondendo a menos de uma a cada mil admissões hospitalares ${ }^{2,3}$, sua letalidade permanece alta, variando entre $30 \%$ e

\footnotetext{
1 Departamento de Hemodinâmica, Cardiologia e Radiologia Vascular Intervencionista - Hospital Madre Teresa - Belo Horizonte, MG, Brasil.

Correspondência: Marcos Antônio Marino. Av. Raja Gabaglia, 1.002 Bairro Gutierrez - Belo Horizonte, MG, Brasil - CEP 30380-090 E-mail: marino@uai.com.br

Recebido em: 10/9/2009 - Aceito em: 27/11/2009
}

\begin{abstract}
Percutaneous Intervention in Mesenteric Arterial Ischemia: Indications, Techniques and Treatment

Scientific reports on mesenteric arterial ischemia suggest that the disease is not thoroughly understood, is poorly diagnosed and rarely treated, despite its high lethality. The prognosis of mesenteric ischemia depends on a fast restoration of blood flow, which may be achieved by clinical, surgical and endovascular management or by a combination of all them. This article reviews the diagnostic evidence of mesenteric ischemia and the available therapeutic options including percutaneous intervention as an alternative method to surgery, which is associated to significant mortality and morbidity rates.
\end{abstract}

DESCRIPTORS: Mesenteric arteries/surgery. Mesenteric arteries/diagnosis. Ischemia/diagnosis. Ischemia/surgery. Angioplasty, balloon. Mesentery/blood supply. Fibrinolytic agents/therapeutic use. Tirofiban/administration \& dosage. Anticoagulants/therapeutic use.

$90 \%$, dependendo da etiologia ${ }^{4,5}$. A isquemia mesentérica está presente principalmente em indivíduos idosos e geralmente naqueles com algum transtorno cardiovascular prévio, como fibrilação atrial ${ }^{6}$.

A isquemia mesentérica resulta do envolvimento de vários fatores, acarretando apresentação clínica diversa e consequente dificuldade em ser diagnosticada. O prognóstico da isquemia mesentérica, por conseguinte, dependerá da detecção oportuna e de uma abordagem específica antes que seus efeitos deletérios se instalem.

O tratamento da isquemia mesentérica é obtido com o restabelecimento do fluxo sanguíneo, seja por meio de tratamento clínico, cirúrgico, endovascular ou da combinação destes. Com o maior entendimento da doença, novas alternativas terapêuticas têm sido desenvolvidas. Na década de 1980, foram relatadas as primeiras experiências com intervenções percutâneas para correção de lesões em vasos mesentéricos e, 
desde então, esses procedimentos têm demonstrado grande potencial, sendo uma alternativa à cirurgia, que é associada a significativas taxas de morbidade e mortalidade ${ }^{7}$.

Esta revisão aborda brevemente os tipos de isquemia arterial mesentérica, sua apresentação clínica e evidência diagnóstica, além de discutir a aplicabilidade da intervenção endovascular percutânea.

\section{CONCEITO E CLASSIFICAÇÃO DA ISQUEMIA MESENTÉRICA}

A isquemia mesentérica ocorre quando a perfusão dos principais órgãos irrigados pela circulação mesentérica (artéria celíaca, artéria mesentérica superior, artéria mesentérica inferior e ramos colaterais), incluindo intestino delgado, intestino grosso, estômago, fígado, vesícula biliar e pâncreas, é insuficiente para supri-los em suas necessidades metabólicas ${ }^{8}$. Essa insuficiência pode ocorrer por mecanismos diferentes, como obstruções arteriais, venosas e da microcirculação, ou mesmo na ausência de obstrução vascular, quando existe um transtorno expressivo da perfusão tecidual, como acontece nos casos de insuficiência cardíaca, choque, desidratação e hipotensão arterial. As lesões intestinais, por sua vez, são consequentes à falta de suprimento sanguíneo (isquemia) e, também, da reperfusão ${ }^{9}$.

A isquemia mesentérica representa uma variedade de distúrbios e talvez seja esse o motivo da dificuldade em classificá-la. Muitos trabalhos classificam-na em aguda e crônica; entretanto, as manifestações clínicas da isquemia mesentérica dependem não apenas da rapidez do processo, mas do envolvimento de outros fatores, tais como $^{10}$ : segmento afetado, vaso envolvido, grau de comprometimento, e etiologia do processo isquêmico.

A isquemia intestinal pode se apresentar como: isquemia do cólon $(60 \%)$, isquemia mesentérica aguda $(30 \%)$, isquemia focal segmentar $(5 \%)$, e isquemia mesentérica crônica (5\%). ${ }^{5,11}$ A colite isquêmica é o tipo mais comum de isquemia mesentérica, com menor letalidade que a forma aguda. As anormalidades patológicas envolvidas na isquemia do cólon são variadas e comumente não há indicação para angiografia mesentérica, pois, por ocasião da apresentação, a circulação do cólon já terá voltado ao normal ${ }^{11,12}$.

\section{ANATOMIA VASCULAR}

O tronco celíaco, a artéria mesentérica superior e a artéria mesentérica inferior são ramos da aorta abdominal em sua face ventral. Em conjunto, proporcionam a maior parte da irrigação do trato gastrointestinal, recebendo cerca de $25 \%$ do débito cardíaco e aumentando seu fluxo durante a digestão. Para prevenção de isquemia existe uma complexa rede de anastomoses entre as principais artérias mesentéricas ${ }^{5,8}$.
O tronco celíaco tem localização superior, originando-se logo abaixo do hiato aórtico, e divide-se em: artéria gástrica esquerda, artéria hepática comum e artéria esplênica. Pode, também, dar origem a uma ou ambas as artérias frênicas inferiores e, mais raramente, à artéria mesentérica superior.

A artéria gástrica esquerda é o menor ramo do tronco celíaco. Superiormente, saem dois ou três ramos esofágicos que se anastomosam com os ramos esofágicos da aorta. A artéria então volta-se ânteroinferiormente, irrigando ambas as faces gástricas, e anastomosa-se com a artéria gástrica direita.

A artéria hepática comum é intermediária em tamanho entre a artéria gástrica esquerda e a artéria esplênica. Estende-se do tronco celíaco até a origem da artéria gastroduodenal, continuando como artéria hepática própria até o ponto de bifurcação. A artéria hepática dá origem às artérias gástrica direita, gastroduodenal e cística, aos ramos para o ducto colédoco, a partir da artéria hepática direita, e, algumas vezes, à artéria supraduodenal.

A artéria esplênica é o maior ramo do tronco celíaco, sendo notavelmente tortuosa. Divide-se em cinco ou mais ramos segmentares terminais antes de penetrar no hilo do baço. Além desses ramos, a artéria esplênica divide-se em ramos pancreáticos, artérias gástricas curtas, artéria gástrica posterior e artéria gastro-omental esquerda.

A artéria mesentérica superior origina-se cerca de $1 \mathrm{~cm}$ abaixo do tronco celíaco e irriga todo o intestino delgado, exceto a parte superior do duodeno, o ceco, o cólon ascendente e a maior parte do cólon transverso. Do lado direito da artéria mesentérica superior originam-se, no sentido craniocaudal, as artérias pancreaticoduodenais inferiores, a artéria cólica média, a artéria cólica direita e a artéria ileocólica. A artéria cólica média divide-se em dois ramos: o direito, que se anastomosa com a artéria cólica direita; e o esquerdo, com a artéria cólica esquerda, um ramo da artéria mesentérica inferior. Os arcos por eles formados irrigam o cólon transverso. A artéria cólica direita origina-se próximo ao meio da artéria mesentérica superior. Ela se divide em um ramo descendente, que se anastomosa com a artéria ileocólica, e um ramo ascendente, que se comunica com a artéria cólica média. Essas anastomoses formam arcos, a partir dos quais os vasos irrigam o cólon ascendente em seus dois terços superiores, incluindo a flexura cólica direita. A artéria ileocólica é o último ramo a partir do lado direito da artéria mesentérica superior. Seu ramo superior anastomosa-se com a artéria cólica direita e seu ramo inferior, com a terminação da artéria mesentérica superior. Do lado esquerdo da artéria mesentérica superior originam-se as artérias jejunais e ileais, em número de 12 a 15. Esses ramos intestinais formam uma série de arcadas (chegando até cinco) antes de 
penetrarem na parede intestinal através das artérias retas. As artérias retas parecem ser terminais e a oclusão seletiva de seu lúmen pode acarretar infarto intestinal segmentar.

A artéria mesentérica inferior é a mais caudal e a menor das três, irrigando o terço esquerdo do cólon transverso, o cólon descendente, o sigmóideo e as porções proximais do reto. A artéria mesentérica inferior possui ramos cólico esquerdo, sigmóideo e retal superior. O ramo ascendente da artéria cólica esquerda anastomosa-se com a cólica média e o ramo descendente, com a artéria sigmóidea mais alta. As artérias sigmóideas, por sua vez, em número de duas ou três, além da anastomose com a cólica esquerda, comunicam-se, através de seu ramo mais inferior, com a artéria retal superior. As artérias sigmóideas, analogamente ao que ocorre na artéria mesentérica superior, fazem uma série de arcadas, que terminam em artérias retas. Já o ramo retal superior é uma continuação da artéria mesentérica inferior. Ele se divide, próximo à terceira vértebra sacral, em dois ramos descendentes, que se dividem novamente para irrigar o reto.

A circulação mesentérica oferece múltiplas possibilidades de colateralização, que podem ser demonstradas angiograficamente. Entre a artéria mesentérica superior e o tronco celíaco, a colateralização se dá principalmente através dos vasos gastroduodenais e pancreaticoduodenais. Já entre a artéria mesentérica superior e a artéria mesentérica inferior, a maior rede de anastomose ocorre através da artéria marginal de Drummond, uma artéria contínua ao cólon, formada por ramos da ileocólica e das cólicas direita, média, esquerda e sigmóidea. A presença de alargada artéria marginal de Drummond à angiografia sugere oclusão de uma das artérias mesentéricas. Nessa situação, outra comunicação que pode ser evidenciada é a arcada de Riolan, geralmente formada por ramos da cólica média e da cólica esquerda.

Além da intercomunicação na própria circulação mesentérica, há importante colateralização com a circulação sistêmica. No território da artéria mesentérica inferior e da artéria ilíaca interna, essa comunicação se dá através das artérias hemorroidárias; já no território do tronco celíaco com aorta descendente, a comunicação ocorre através das artérias frênicas ${ }^{8}$.

Nas áreas nas quais há abundante circulação colateral, como o duodeno e o reto, a isquemia é rara. Todavia, em regiões como o ângulo esplênico (ponto de Griffiths) e a flexura retossigmóidea (ponto de Sudeck) a circulação colateral existe, porém é mais deficiente, o que confere risco mais alto para isquemia.

Decerto, o suprimento sanguíneo da porção intraabdominal do trato gastrointestinal é ricamente dotado de intercomunicações e anastomoses, que ajudam a protegê-lo. No entanto, se essa verdadeira rede se tornar insuficiente, seja por um processo obstrutivo seja por uma condição que leve ao baixo fluxo nesse território vascular, ocorre a isquemia mesentérica.

\section{APRESENTAÇÃO CLÍNICA E DIAGNÓSTICO DA ISQUEMIA ARTERIAL MESENTÉRICA}

O diagnóstico de isquemia intestinal é frequentemente realizado pela exclusão de afecções mais comuns. O paciente pode se queixar de dor abdominal isolada ou associada a outros sintomas, como náuseas, vômitos e diarreia'13; entretanto, tais queixas representam um quadro clínico amplo e que pode estar presente em outros distúrbios do trato gastrointestinal, como obstrução intestinal, complicações da litíase biliar, diverticulite ou, simplesmente, gastroenterite ${ }^{3}$. Na avaliação laboratorial de rotina são solicitados hemograma, bioquímica do soro, coagulograma, gasometria arterial, amilase, lípase, ácido láctico, tipo sanguíneo, prova cruzada e enzimas cardíacas. Todavia, alterações nesses exames não são específicas para isquemia mesentérica ${ }^{11,13}$. Informações adicionais podem ser obtidas por meio de métodos de imagem, como ultrassonografia com ecocardiograma Doppler bidimensional, tomografia computadorizada, ressonância nuclear magnética e angiografia.

\section{Isquemia mesentérica aguda oclusiva}

A isquemia mesentérica aguda oclusiva desenvolve-se em minutos a horas em decorrência de oclusão súbita das artérias que suprem o intestino, levando a perfusão insuficiente para sua viabilidade. Entre $70 \%$ e $90 \%$ dos portadores de isquemia mesentérica aguda oclusiva morrem, apesar de cirurgia ou terapia endovascular imediatas. Os sobreviventes, não raro, convivem com dificuldades advindas de grandes ressecções de alças intestinais, necessitando de alimentação parenteral ou enteral ${ }^{14,15}$.

As principais causas de oclusão arterial aguda mesentérica incluem, principalmente, embolia (50\%) e trombose $(25 \%)^{16}$. A embolia das artérias mesentéricas ocorre predominantemente no território da artéria mesentérica superior, em decorrência de seu calibre maior e de sua obliquidade ao se originar da aorta. Na maioria das vezes, a oclusão embólica da artéria mesentérica superior ocorre distalmente a sua origem, até um ponto no qual a ramificação reduz seu lúmen para um diâmetro menor que o do êmbolo. Geralmente esse ponto é a divisão da artéria cólica média e dos ramos jejunais ${ }^{17}$. Já a trombose das artérias mesentéricas comumente é uma complicação da aterosclerose prévia. Ocorre usualmente na origem do vaso, adjacente à doença aterosclerótica ostial. A artéria mesentérica superior é a mais afetada, seguida do tronco celíaco e da artéria mesentérica inferior ${ }^{5,18}$.

O infarto intestinal está associado a alta morbidade, o que se deve em especial ao fato de o leito vascular mesentérico ainda ser relativamente pobre em colaterais 
(quadro agudo) e ao fato de que o diagnóstico nem sempre é realizado facilmente. Para que ocorram sintomas é preciso ao menos dois dos vasos mesentéricos ocluídos e estenose em um terceiro, embora existam casos na literatura de isquemia mesentérica aguda originada de lesão crítica de apenas um dos vasos. Pacientes que têm sua rede de colaterais subitamente ocluída após realização de alguma cirurgia abdominal também podem apresentar quadro de isquemia mesentérica aguda.

Os principais indícios para diagnóstico da isquemia mesentérica aguda oclusiva são ${ }^{19}$ :

- pacientes com dor abdominal aguda fora de proporção em relação ao exame físico e que tenham história pregressa de doença cardiovascular (classe I, nível de evidência B);

- pacientes que tenham desenvolvido dor abdominal aguda após intervenções arteriais nas quais o cateter tenha atravessado a aorta abdominal ou qualquer artéria próxima ou, ainda, que tenham desenvolvido arritmia (como fibrilação atrial), bem como recente isquemia mesentérica (classe I, nível de evidência C).

Aproximadamente dois terços dos pacientes com isquemia intestinal aguda são mulheres na faixa dos 70 anos, doença cardiovascular está presente na maioria dos pacientes, e dor abdominal está sempre presente, apesar de seu tipo, localização e duração serem variáveis. Sua localização mais comum é periumbilical e muita intensa, a ponto de chamar a atenção médica imediata. Inicialmente, sinais de irritação peritoneal estão ausentes e classicamente é uma "dor fora de proporção com os achados do exame físico". Dentre os exames laboratoriais, deve-se lembrar que a presença de acidose é um indicador importante de presença de intestino necrosado.

A angiografia das mesentéricas nas projeções ântero-posterior e lateral é o método que mais auxilia o diagnóstico de isquemia mesentérica aguda oclusiva, sendo considerado padrão de referência. Todavia, seu uso deve ser individualizado, haja vista o tempo requerido para sua realização e o risco iminente de vida. Em pacientes com suspeita de isquemia mesentérica aguda oclusiva, a arteriografia mesentérica pode auxiliar na diferenciação entre isquemia oclusiva e não-oclusiva, evitando, nessa situação, cirurgia desnecessária. Nos pacientes cuja apresentação clínica é mais demorada ou há alta probabilidade de isquemia não-oclusiva, a arteriografia inicial é indicada, já que a efetividade do método superaria o risco (classe I, nível de evidência B).

Apesar de a ultrassonografia com ecocardiograma Doppler bidimensional ser capaz de identificar lesões oclusivas nas artérias intestinais, na prática ela não auxilia muito. A distensão abdominal e os fluidos frequentemente presentes na isquemia mesentérica agu- da impossibilitam o sucesso de tal método. Por causa da emergência no tratamento, esse exame é contraindicado (classe III, nível de evidência C). Como na tomografia computadorizada há necessidade de se administrar contraste iodado intravenoso, que poderá afetar a acuidade de uma angiografia sequencial, esse método não é indicado inicialmente, embora seja realizado com frequência antes que se pense no diagnóstico de isquemia mesentérica aguda oclusiva ${ }^{15}$.

A despeito dos exames de imagem, em pacientes com suspeita de infarto intestinal a laparotomia para ressecção da região inviável não deve ser adiada.

\section{Isquemia mesentérica aguda não-oclusiva}

A forma não-oclusiva corresponde a $20 \%$ das causas de isquemia mesentérica aguda. Apesar de não haver obstrução fixa, o vasoespasmo nas artérias mesentéricas pode ocasionar infarto do intestino. As condições que levam a suspeitar de isquemia mesentérica aguda não-oclusiva são:

- pacientes em estado de choque, sobretudo cardiogênico, que desenvolvam dor abdominal (classe I, nível de evidência B);

- pacientes em uso de substâncias ou medicamentos vasoconstritores, como cocaína, derivados do ergot, vasopressina ou norepinefrina, que desenvolvam dor abdominal (classe I, nível de evidência B);

- pacientes que desenvolvam dor abdominal após reparo de coarctação da aorta ou após revascularização de isquemia intestinal por obstrução arterial (classe I, nível de evidência $B$ ).

Apesar dessas recomendações, não existem dados do exame físico ou testes laboratoriais específicos para isquemia mesentérica aguda não-oclusiva. O estudo angiográfico mesentérico também é o método diagnóstico padrão de referência nessa situação. Esse exame pode demonstrar as características do vasoespasmo arterial mesentérico, além de indicar a administração intra-arterial de medicamento vasodilatador, como papaverina. O estudo angiográfico mesentérico é indicado na suspeita de isquemia mesentérica aguda nãooclusiva, desde que se esteja tratando a causa básica do vasoespasmo e a evolução do processo não seja insidiosa (classe I, nível de evidência B).

\section{Isquemia mesentérica crônica}

A isquemia mesentérica crônica corresponde a apenas $5 \%$ das isquemias mesentéricas. A aterosclerose é a principal causa de redução de fluxo nas artérias do leito mesentérico e a presença de lesões significativas é observada entre $6 \%$ e $10 \%$ das autópsias e em $14 \%$ a $24 \%$ dos pacientes submetidos a arteriografia. Apesar de a doença aterosclerótica desses vasos ser comum, a manifestação clínica da isquemia intestinal crônica é rara. Esse pacientes, em sua maioria, são idosos e predominantemente do sexo feminino. 
Alguns relatos para o diagnóstico de isquemia mesentérica crônica têm sugerido, com certa frequência, que essa síndrome requer a oclusão de pelo menos duas das três artérias; entretanto, esse conceito não é inteiramente correto $^{20}$. Existem relatos de casos bem documentados de isquemia mesentérica crônica na ocorrência da oclusão de um único vaso, a artéria mesentérica superior. Pacientes submetidos a cirurgia de ressecção de intestino ou reparo de aneurisma de aorta são mais vulneráveis a isquemia por obstrução de vaso único.

A isquemia mesentérica crônica é caracterizada classicamente por dor abdominal ou angina intestinal, que se agrava com a alimentação. Essas manifestações clínicas costumam ocorrer cerca de 30 minutos após a refeição, perdurando por uma a três horas, período no qual o consumo de oxigênio é aumentado pela digestão. Além da dor, a perda de peso, decorrente da diminuição da ingesta alimentar, é também uma condição associada. Vômitos, diarreia e constipação estão presentes em uma minoria de pacientes. Em 2005, Brown et al. ${ }^{21}$ publicaram um estudo com pacientes tratados de isquemia mesentérica crônica com implante de stent. Todos os pacientes apresentaram dor abdominal, $71 \%$ apresentaram perda de peso (média de perda: $11 \mathrm{~kg}$ ) e $57 \%$ relataram medo de se alimentar. Esses dados clínicos ilustram os principais indícios que levam à suspeita de isquemia mesentérica crônica: dor abdominal e perda de peso sem outra explicação, sobretudo se concomitante a doença cardiovascular (classe I, nível de evidência B).

No entanto, como existem inúmeras causas de dor abdominal acompanhada de perda de peso, e porque a isquemia mesentérica crônica é uma doença rara, o diagnóstico de isquemia mesentérica crônica quase sempre é atrasado. Atualmente, não há testes que estabeleçam o diagnóstico definitivo, mas é a combinação entre a apresentação clínica e o achado de obstrução arterial mesentérica na ausência de outra causa óbvia que leva ao diagnóstico. A ultrassonografia com ecocardiograma Doppler bidimensional dos vasos viscerais pode auxiliar o diagnóstico de isquemia mesentérica crônica. Apesar de ser tecnicamente difícil, o exame pode ser realizado em mais de $85 \%$ dos pacientes eletivos, tendo acurácia de até $90 \%$ na detecção de estenoses significativas (igual ou superior a 70\%) da artéria celíaca e/ou da artéria mesentérica superior ${ }^{22}$. Usando essa técnica, Hansen et al. ${ }^{23}$ encontraram prevalência de $17,5 \%$ de estenoses acima de $70 \%$ em indivíduos com mais de 65 anos. A tomografia computadorizada contrastada e a ressonância nuclear magnética com gadolínio, por sua vez, embora sejam bons métodos para visualização de lesões ateroscleróticas na origem das artérias, apresentam baixa acurácia para visualização de lesões distais e para o diagnóstico de causas menos comuns de isquemia mesentérica. Esses três exames são inicialmente válidos se somados às evidências clínicas para identificação da isquemia mesentérica crônica (classe I, nível de evidência B).

A aortografia em dois planos com incidência lateral, decerto, é essencial para demonstrar lesões típicas (aquelas que ocorrem na região ostial do vaso), devendo ser realizada em pacientes com suspeita de isquemia mesentérica crônica nos quais os métodos não-invasivos foram inconclusivos (classe I, nível de evidência B). Ademais, esse método pode revelar lesões únicas na parte proximal não-ostial, que são menos frequentes.

A aortografia é o método que pode explorar melhor as variações anatômicas relevantes para intervenção. Ao contrário dos casos agudos, na isquemia crônica observa-se a presença de vasta rede de colaterais:

- ramos intercelíacos: arco gástrico e arco gastroepiploico;

- tronco celíaco e artéria mesentérica superior: arco pancreático;

- artérias mesentérica superior e inferior: arco de Riolan (vaso medial da artéria cólica esquerda), artérias marginais de Drummond (ramos mediais e da cólica esquerda);

- artéria mesentérica inferior e artérias ilíacas: arco retal (hemorroidário).

A presença de alargada arcada de Riolan é um sinal arteriográfico de obstrução arterial mesentérica superior ou inferior, estabelecendo, portanto, a circulação colateral.

A aortografia pode também diferenciar entre estenoses proximais do tronco celíaco e compressões extrínsecas do troco celíaco. A causa mais frequente dessa condição é o cruzamento do ligamento arqueado mediano do diafragma mais baixo que o habitual (síndrome do ligamento arqueado mediano). Sabe-se, porém, que a compressão visualizada pela aortografia é, na maioria das vezes, assintomática.

\section{OPÇÕES TERAPÊUTICAS PARA OS PACIENTES COM ISQUEMIA ARTERIAL MESENTÉRICA}

\section{Isquemia mesentérica aguda oclusiva}

A terapêutica de escolha da isquemia mesentérica aguda oclusiva varia conforme a etiologia do processo, podendo associar medidas essencialmente clínicas, procedimentos endovasculares percutâneos e cirurgia. Para os pacientes com apresentação muito aguda, a melhor abordagem é a laparotomia imediata por um cirurgião capaz de realizar a revascularização.

\section{Tratamento cirúrgico}

O tratamento cirúrgico da isquemia mesentérica aguda oclusiva consiste em laparotomia, revascu- 
larização do intestino isquêmico, ressecção de áreas do intestino necrosadas e avaliação da viabilidade do intestino após a revascularização. A técnica de revascularização depende da etiologia da isquemia mesentérica aguda oclusiva. Uma vez confirmado o diagnóstico de embolia, a embolectomia deve ser imediata. Já o tratamento da trombose arterial aguda se dá por tromboendarterectomia ou pela realização de enxertos vasculares. Após o procedimento o paciente requer cuidados intensivos. Durante a intervenção, podese programar uma operação de revisão (second look) 24 a 48 horas após o procedimento inicial, para reavaliação da viabilidade intestinal, evitando tanto a ressecção excessiva quanto a falha na ressecção de intestino não-viável. Essas etapas, portanto, constituem o tratamento cirúrgico da isquemia intestinal aguda (classe I, nível de evidência B).

\section{Tratamento endovascular}

Apesar da limitação de dados, o tratamento percutâneo da obstrução arterial é uma medida sensata, dada a grande mortalidade associada à abordagem cirúrgica padrão. Todavia, como a maioria dos pacientes portadores de isquemia mesentérica aguda oclusiva tem alguma parte de intestino inviável, vários desses pacientes necessitarão de laparotomia e acesso cirúrgico para avaliação de viabilidade intestinal, mesmo que a intervenção percutânea obtenha sucesso angiográfico. O restabelecimento de fluxo para o intestino infartado causa liberação súbita de endotoxinas, que se associam a coagulação intravascular disseminada, síndrome do desconforto respiratório do adulto e colapso cardiovascular ${ }^{11,13,19}$. Portanto, na presença de intestino infartado ou de níveis elevados de ácido láctico, o tratamento percutâneo inicial deve ser ponderado em relação às opções de tratamento cirúrgico que podem controlar o fluxo venoso e as endotoxinas que seriam liberadas do segmento que sofreu isquemia. Assim, a intervenção percutânea na isquemia mesentérica aguda oclusiva, incluindo terapia lítica transcateter, angioplastia com balão e colocação de stent, se aplica em pacientes selecionados (Figuras 1 e 2). Tais pacientes também necessitam de laparotomia de revisão. Segundo o American College of Cardiology/American Heart Association (ACC/AHA), essa conduta tem eficácia menos estabelecida, sendo baseada apenas em relatos de casos ou opiniões de especialistas (classe Ilb, nível de evidência C).

\section{Terapia endovascular vs. cirurgia}

Para pacientes com apresentação superaguda, alta probabilidade de obstrução arterial e suspeita de intestino necrosado, a laparotomia de urgência para revascularização intestinal, quando realizada por cirurgião experiente, parece ser a melhor opção. A mortalidade nesses casos é de cerca de $70 \%$. A revascularização poderá ser realizada tanto por embolectomia como por enxerto. O intestino necrosado pode ser ressecado e uma nova laparoscopia pode ser programada entre 24 e 48 horas após a primeira.

$\mathrm{Na}$ presença de isquemia aguda, a abordagem endovascular pode ser utilizada para restauração do fluxo naqueles pacientes com risco cirúrgico proibitivo, particularmente se não forem observados sinais de peritonite.

A opção pela terapia endovascular traz como opções a possibilidade de se realizar infusão de medicações trombolíticas, como abciximab e/ou tirofibam, diretamente no sítio de obstrução intra-arterial ou, ainda, extrair o trombo por sucção ou lise mecânica.

As principais dificuldades encontradas estão em se determinar qual o vaso responsável pela isquemia aguda, e se não estaríamos diante de obstrução total crônica. Outras dificuldades podem ser encontradas, como:

- dissecção na parede subintimal;

- embolização para os ramos;

- necessidade de guia com bom suporte.

\section{Isquemia mesentérica aguda não-oclusiva}

O tratamento inicial da isquemia intestinal nãooclusiva está diretamente relacionado ao tratamento da causa base do vasoespasmo, comumente desencadeado por choque circulatório. A maneira mais segura de recuperação do vasoespasmo arterial é pela monitorização hemodinâmica, incluindo terapia farmacológica e de reposição de volume a fim de melhorar o débito cardíaco e a perfusão periférica. A abordagem inicial do choque é a medida mais importante no tratamento da isquemia mesentérica aguda não-oclusiva (classe I, nível de evidência C). A administração de vasodilatadores através de cateter por via percutânea no sítio vascular em vasoespasmo tem se associado a alívio dos sintomas em vários pacientes ${ }^{24}$. Tal conduta é especialmente apropriada em isquemia não-oclusiva causada por uso de substâncias como cocaína e derivados do ergot, nos quais não há coexistência com choque circulatório (classe lla, nível de evidência B). Caso os sintomas abdominais persistam após alívio do vasoespasmo há necessidade de laparotomia para definir a vitalidade intestinal e a ressecção do intestino necrótico (classe I, nível de evidência B).

\section{Isquemia mesentérica crônica}

\section{Tratamento cirúrgico}

O tratamento cirúrgico da isquemia mesentérica crônica é realizado por endarterectomia transaórtica ou enxerto, com preferência pelo último. Em geral, o acompanhamento clínico dos pacientes mostra alívio dos sintomas com poucas recorrências. Mesmo assim, o acompanhamento é necessário. A recorrência dos sintomas, na maioria das vezes, é resultado de estenose 


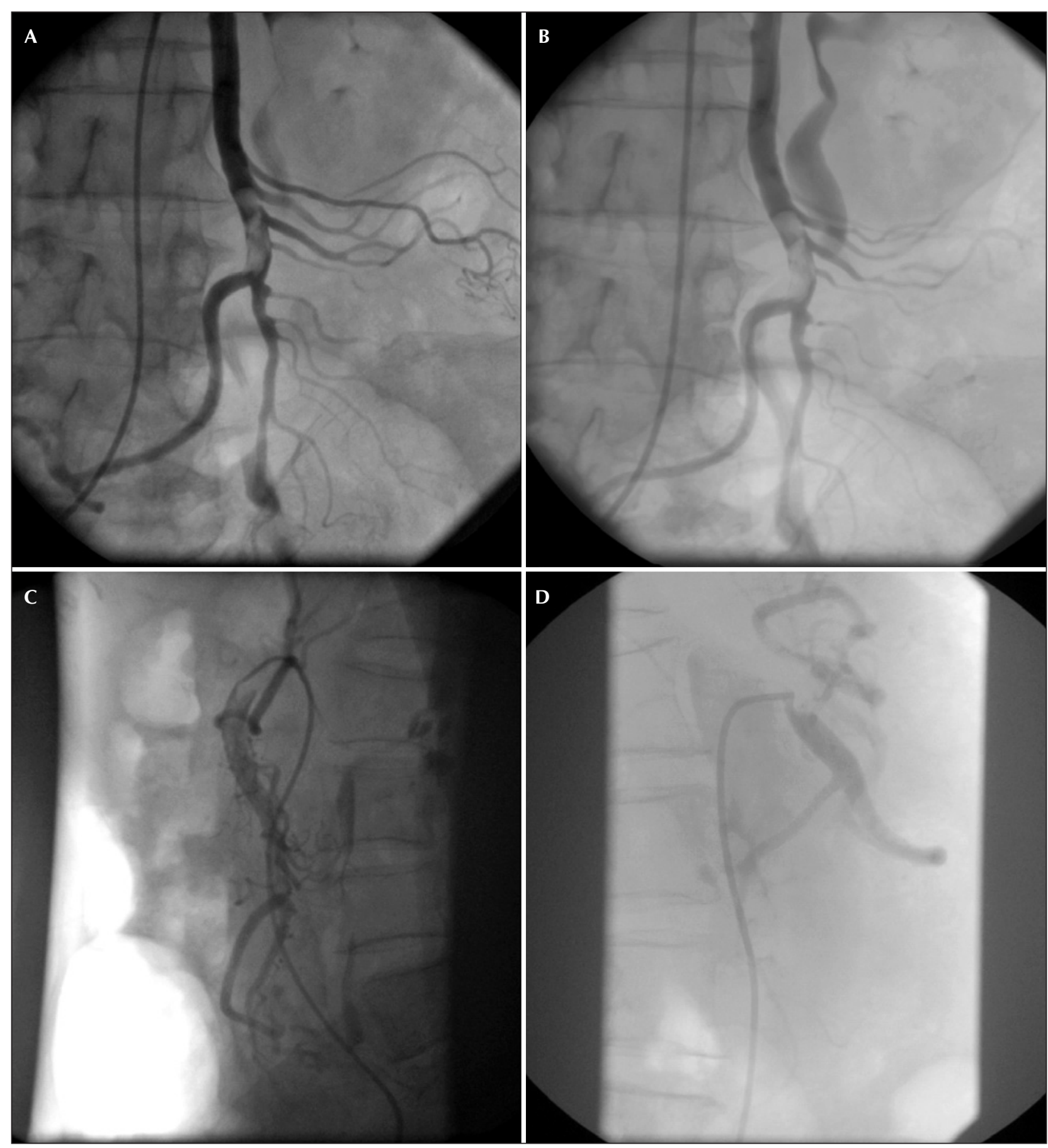

Figura 1 - Paciente com 80 anos de idade, com colectomia prévia por megacólon chagásico e acidente vascular cerebral prévio, em uso de digoxina e ácido acetilsalicílico, apresentando acidente isquêmico transitório e dor periumbilical, constante e abrupta, acompanhada de um episódio de vômito. Em A, B e C, presença de trombo no terço médio da artéria mesentérica superior. Em D, tronco celíaco evidenciando estenose excêntrica grave de $90 \%$ na região ostial.

das artérias viscerais ou de reestenose do sítio revascularizado. Tradicionalmente o tratamento da isquemia mesentérica crônica é cirúrgico (classe I, nível de evidência B). Em estudo que representa a evolução de pacientes com isquemia mesentérica crônica tratados cirurgicamente, demonstrou-se que cinco anos após o procedimento a perviedade da reconstrução arterial permaneceu entre $57 \%$ e $69 \%$. Ademais, a ausência de remissão dos sintomas em cinco e em dez anos foi de cerca de $80 \%$ e $60 \%$, respectivamente ${ }^{25}$. 


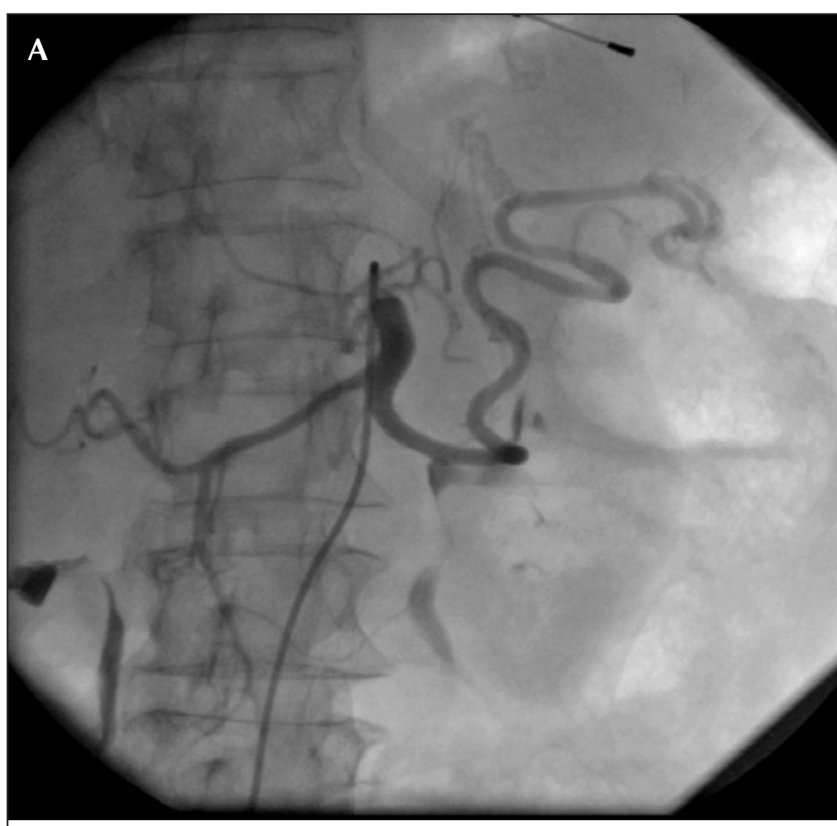

B

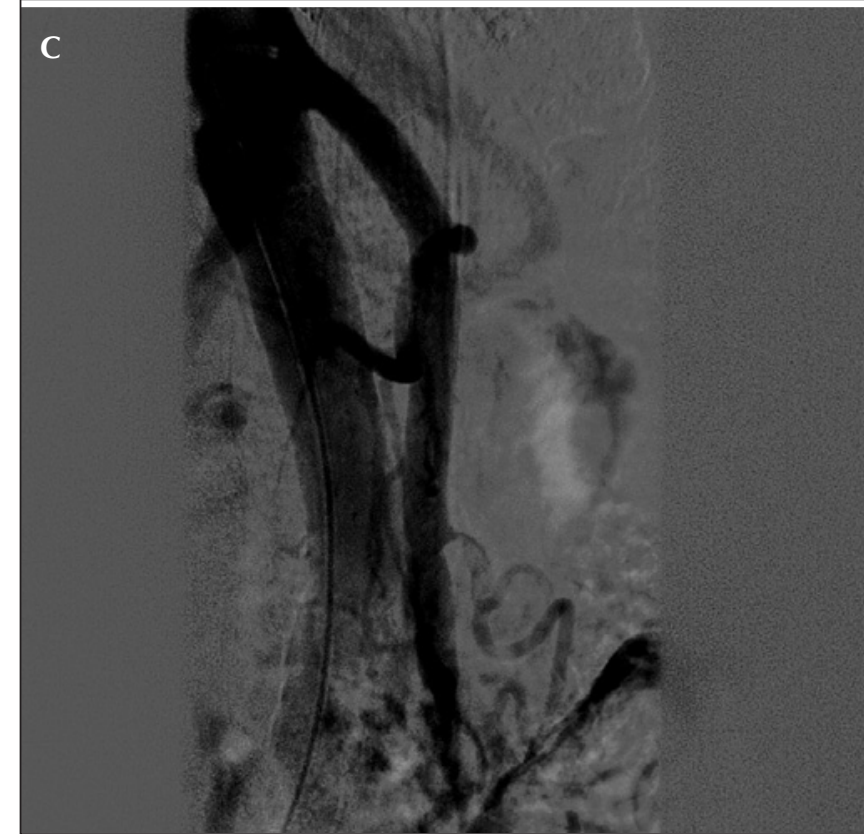

D
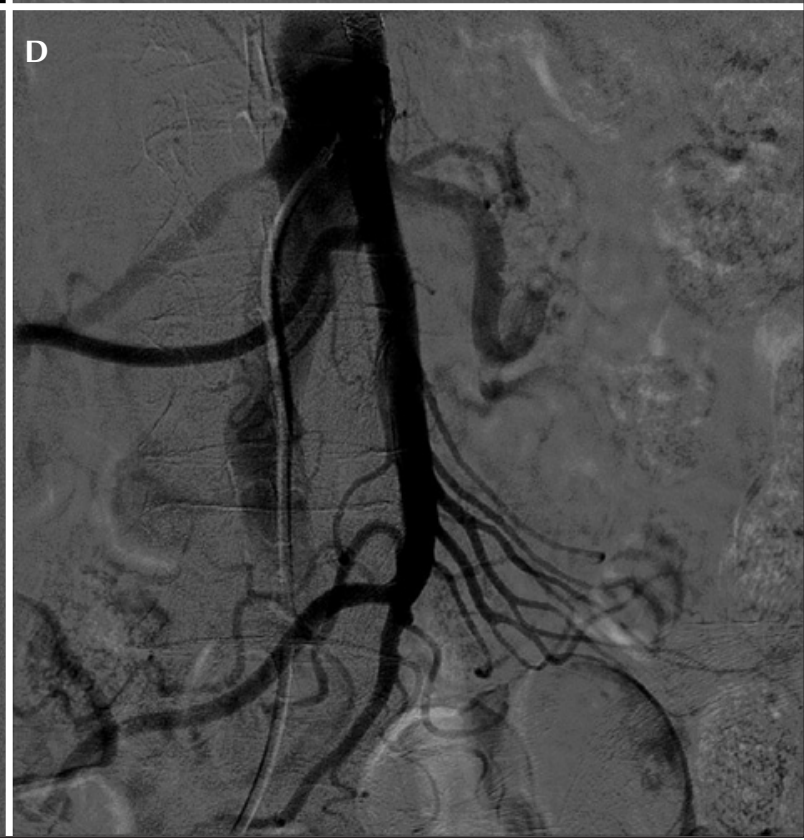

Figura 2 - Realizada heparinização plena (5000 UI) e bolus de tirofibam diretamente na artéria mesentérica superior, com manutenção de heparina e tirofibam por cinco dias. Em A e B, resultado após angioplastia do tronco celíaco com implante de stent Dinamic 7 x 12 mm. Em C e D, artéria mesentérica superior isenta de trombos ou qualquer lesão residual.

Outra condição na qual a revascularização cirúrgica pode ser considerada é a observação de obstrução arterial assintomática em pacientes submetidos a cirurgia aórtica ou renal por outras indicações. No entanto, a efetividade dessa conduta é menos estabelecida (classe Ilb, nível de evidência B). Assim, excetuando-se os casos de cirurgia aórtica ou renal, a revascularização cirúrgica é contraindicada em pacientes assintomáticos, podendo até ser nociva (classe III, nível de evidência B).
Com o progresso na intervenção percutânea, esse procedimento tem sido cada vez mais empregado no tratamento da isquemia mesentérica crônica. Sua aplicabilidade na intervenção da isquemia mesentérica crônica será discutida a seguir.

\section{Tratamento endovascular}

Desde o primeiro relato sobre a aplicação da angioplastia no tratamento da isquemia mesentérica crônica, grande número de trabalhos tem documenta- 
do a correção da obstrução arterial intestinal, por essa técnica, com grande êxito. A primeira vantagem do tratamento endovascular é ser pouco invasivo, não requerendo anestesia geral, laparotomia ou grandes procedimentos operatórios, além de, usualmente, reduzir significativamente os dias de hospitalização no período pós-operatório.

Revascularizações abertas são procedimentos complexos, de elevadas morbidade e mortalidade, sobretudo em pacientes idosos. A angioplastia transluminal percutânea por balão e/ou stent tem sido indicada como alternativa à intervenção cirúrgica na isquemia mesentérica crônica (classe I, nível de evidência B).

Assim como a cirurgia é a opção mais frequente na vigência de quadro agudo, a angioplastia vem crescendo em preferência nas apresentações crônicas. O tratamento das lesões deve ser realizado em pacientes sintomáticos (Figura 3), de forma profilática em pacientes com estenose mesentérica que irão realizar cirurgia abdominal eletiva (risco de perder artérias colaterais ou fenômeno de roubo de fluxo), e deve ter como objetivos o alívio dos sintomas, a melhora da condição nutricional e a prevenção do infarto intestinal.

Revendo a literatura recente, nota-se tanto escassez de estudos prospectivos terapêuticos como limitação no seguimento clínico desses pacientes. Vários relatos de série de casos tratados por angioplastia com balão e/ou stent indicam maior recorrência dos sintomas após essa conduta. Contudo, em muitas das recorrências foi possível reintervir, pelo mesmo método.

Allen et al. ${ }^{26}$ avaliaram pacientes de elevado risco cirúrgico, portadores de isquemia mesentérica crônica, submetidos a angioplastia. Foram tratados por essa técnica 19 pacientes, totalizando 24 estenoses. A taxa de sucesso foi de 95\%. Um paciente evoluiu para óbito após dissecção da artéria mesentérica superior, que resultou em trombose e infarto do intestino; 15 pacientes $(79 \%)$ relataram alívio completo dos sintomas, mantido, em média, por 39 meses; e nos demais pacientes, houve melhora parcial dos sintomas após procedimento, embora nos 28 meses subsequentes houvesse remissão dos sintomas. Os autores concluíram que a angioplastia mesentérica é um procedimento com elevada taxa de sucesso, promovendo imediata melhora dos sintomas num curto intervalo de seguimento ${ }^{26}$.

Em 2002, Matsumoto et al. ${ }^{27}$ publicaram dados retrospectivos de pacientes tratados com angioplastia com balão e/ou stent para isquemia mesentérica crônica. Foram acompanhados 21 mulheres e 12 homens, com média de idade de 63 anos (mínimo 40 e máximo 89 anos). Todos possuíam lesões estenóticas que foram tratadas com angioplastia por balão (21 pacientes, 32 vasos) ou com pré-dilatação e colocação de stent (12 pacientes, 15 vasos). O sucesso técnico da angioplastia com balão e do stent foi de $81,3 \%$ e $100 \%$, respectivamente $(P=0,073)$. Completo alívio dos sintomas ocorreu em $82 \%$ dos pacientes. Em $6 \%$ houve melhora parcial dos sintomas e em $12 \%$ o tratamento foi falho. A média de seguimento nos pacientes que obtiveram sucesso no tratamento foi de 38 meses. A sobrevida em cinco anos pós-procedimento foi de $76,1 \%$ e complicações importantes ocorreram em 13\% dos casos. Ademais, não ocorreram mortes no intervalo de 30 dias. Os autores concluíram que a terapia endovascular para isquemia mesentérica crônica é uma alternativa efetiva à revascularização cirúrgica ${ }^{27}$.

Em artigo de 2006, Silva et al. ${ }^{28}$ divulgaram dados de 59 pacientes submetidos a colocação de stent em artérias mesentéricas com lesões crônicas. Foram revascularizadas 79 artérias, todas com mais de $70 \%$ de estenose. Os pacientes foram acompanhados clinicamente, $90 \%$ deles por meio de métodos propedêuticos não-invasivos (tomografia computadorizada, ultrassonografia) durante seis meses. Sucesso angiográfico foi obtido em $96 \%$ das artérias tratadas e alívio dos sintomas, em 50 pacientes (88\%). Durante o acompanhamento médio de 38 meses, $79 \%$ dos pacientes permaneceram sem sintomas e apenas $17 \%$ relataram remissão dos sintomas. Nesses pacientes foi observada reestenose intrastent, revascularizada com sucesso. Os autores do trabalho consideraram que a angioplastia com stent é efetiva e segura para o tratamento de isquemia mesentérica crônica, alcançando sucesso terapêutico com poucas complicações. Além disso, o alívio dos sintomas é similar ao obtido por tratamento cirúrgico, porém sem as elevadas morbidade e mortalidade associadas a esse procedimento ${ }^{28}$.

Além das séries de casos acerca do tratamento endovascular, existem trabalhos comparando esse tratamento à revascularização cirúrgica. Kasirajan et al. ${ }^{29}$ analisaram a segurança e a eficácia de ambas as técnicas no tratamento de isquemia mesentérica crônica. Para tanto, compararam 28 pacientes com sintomas compatíveis com isquemia mesentérica crônica tratados por angioplastia (balão 5/stent 23) com relatos históricos de pacientes tratados por cirurgia, a maioria por enxerto. Os pacientes apresentavam condições clínicas semelhantes, diferindo quanto à idade: média de 72 anos para os tratados com angioplastia e de 62 anos para os submetidos a cirurgia $(P \leq 0,005)$. Não foram observadas diferenças estatisticamente significantes quanto a complicações intra-hospitalares, mortalidade imediata ou tempo de internação pós-procedimento. A mortalidade após três anos de procedimento foi semelhante, entretanto a recorrência dos sintomas nesse tempo foi maior nos pacientes submetidos a angioplastia ${ }^{29}$.

Analogamente, Sivamurthy et al. ${ }^{30}$ analisaram retrospectivamente pacientes portadores de isquemia mesentérica crônica tratados por angioplastia ou cirurgia no período de 1989 a 2003. Houve equivalência quanto a mortalidade em 30 dias e em três anos em ambos os grupos de pacientes. No entanto, no grupo 


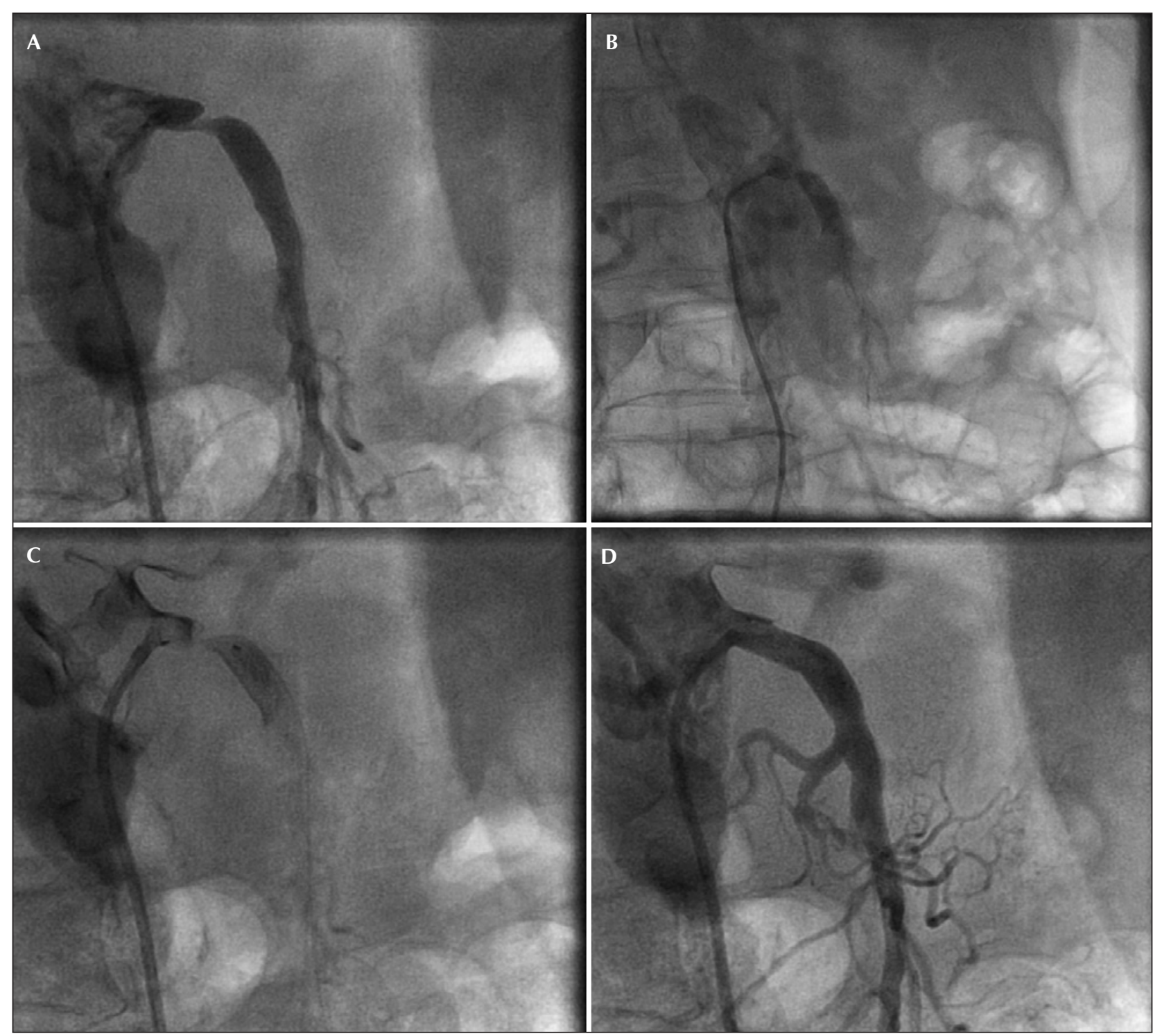

Figura 3 - Paciente portador de vasculopatia periférica, encaminhado para arteriografia dos membros inferiores, relatando dor abdominal de forte intensidade há dois meses, de caráter progressivo e com piora após alimentação. Em A e B, constatada, durante o procedimento, lesão grave na artéria mesentérica superior, com hipofluxo distal. Em C e D, angioplastia de artéria mesentérica superior com implante de stent Genesis Palmaz $6 \times 12 \mathrm{~mm}$, com sucesso.

cirúrgico observou-se maior morbidade $(P \leq 0,001)$ e maior tempo de internação pós-operatório $(P \leq 0,001)$. Por outro lado, o grupo tratado por angioplastia apresentou maior recorrência dos sintomas $(P \leq 0,001)$ no período de seis meses $^{30}$.

Em junho de 2008, Zerbib et al. ${ }^{31}$ publicaram seus resultados obtidos entre 2000 e 2006, comparando os principais eventos clínicos dos tratamentos discutidos. Do total de 29 pacientes com história clínica de isquemia mesentérica crônica, 14 foram submetidos a angioplastia e 15, a revascularização aberta. Os pacientes do primeiro grupo eram significativamente mais idosos $(75,4 \pm 10,1$ anos vs. 57,3 \pm $14,4$ anos, respectivamente; $P=0,0009)$. No grupo tratado por via percutânea, as morbidades cardiovasculares eram significativamente maiores que no grupo cirúrgico $(P=0,04)$. Não foram observadas diferenças entre complicações, mortalidade, tempo de estadia hospitalar ou recorrência de sintomas. Reestenose ocorreu apenas nos pacientes tratados por angioplastia, no entanto essa diferença não foi estatisticamente significante. Dessa forma, os autores consideram a angioplastia o método preferencial em pacientes mais idosos incapazes de serem submetidos a cirurgia ${ }^{31}$.

\section{EXPERIÊNCIA EM NOSSA INSTITUIÇÃO}

O Hospital Madre Teresa é um centro de saúde filantrópico, terciário, referência no tratamento das 
doenças cardiovasculares de alta complexidade da região metropolitana de Belo Horizonte (MG). No período de janeiro de 1994 a maio de 2009, foram registrados 18 casos de isquemia mesentérica (10 agudos e 8 crônicos). A média de idade dos pacientes foi de 70 anos, sendo a maioria $(72,2 \%)$ do sexo feminino.

A mortalidade dos pacientes com isquemia mesentérica aguda oclusiva foi de $80 \%$ e a abordagem terapêutica inicial foi por cirurgia aberta em 3 casos, angioplastia com implante de stent em 6 casos e tratamento clínico em 1 paciente. Os dois únicos pacientes com isquemia mesentérica aguda oclusiva e que obtiveram sucesso no tratamento foram abordados por via endovascular. Já nos pacientes portadores de isquemia mesentérica crônica, foi realizada angioplastia, sendo 7 com implante de stent e 1 com balão. Todos os procedimentos obtiveram sucesso.

\section{Técnica de intervenção}

A técnica de intervenção realizada é semelhante à da angioplastia renal. Utiliza-se introdutor $6 \mathrm{~F}$, seja o acesso braquial ou femoral. Nossa preferência é pelo último, com exceção dos casos em o paciente apresenta doença arterial periférica em membros inferiores ou nos casos em que o óstio da artéria apresenta angulação que impossibilita sua cateterização.

Cateteres-guia como o cateter renal double curved (RDC), JR4, multipurpose e de mamária interna são os mais utilizados, quando realizado acesso femoral. Já pelo acesso braquial, o cateter de escolha é o multipurpose.

Stents coronários ou renais podem ser utilizados. Os stents renais são reservados para os vasos de maior calibre.

\section{Terapia medicamentosa}

Os antiagregantes plaquetários devem ser iniciados entre 3 e 5 dias antes de uma intervenção programada.

Aspirina, em dose de ataque de $300 \mathrm{mg} /$ manutenção de $100 \mathrm{mg} / \mathrm{dia}$, e clopidogrel, em dose de ataque de 300 mg ou 600 mg/manutenção de 75 mg/dia, compõem a terapia antiagregante plaquetária utilizada. A dose de manutenção do clopidogrel deve ser prescrita por pelo menos 30 dias e a aspirina, por tempo indeterminado.

A heparina, na dose de 2.500-5.000 UI, é administrada durante a intervenção, devendo-se atentar para a função renal desses pacientes, minimizando o risco de sangramento.

Antiplaquetários endovenosos, como tirofiban e abciximab, ficam a critério do hemodinamicista, assim como a opção de se realizar bolo intra-arterial dessas drogas durante a intervenção, que são administradas quando se observa presença de trombos ou fluxo reduzido.

\section{CONCLUSÕES}

A interrupção da perfusão sanguínea mesentérica pode resultar em isquemia e necrose intestinal, diagnóstico difícil de estabelecer mas que é imperativo para o manejo adequado do paciente. Assim, o fator determinante para obtenção de sucesso no tratamento é o diagnóstico precoce.

Ademais, é importante compreender que a isquemia mesentérica aguda e a isquemia mesentérica crônica são entidades distintas, sendo de fundamental importância o conhecimento da anatomia e da fisiopatologia dessas formas da doença. $\mathrm{Na}$ isquemia mesentérica aguda oclusiva, o prognóstico é reservado, permanecendo a cirurgia como método de tratamento padrão. No entanto, cada vez mais a abordagem percutânea tem sido considerada em pacientes selecionados. $\mathrm{Na}$ forma crônica da isquemia mesentérica, a intervenção percutânea é uma opção muito atraente, com taxas de sucesso e de sobrevivência comparáveis aos resultados cirúrgicos, e riscos de complicação menores.

Apesar do grande avanço desde as primeiras intervenções percutâneas mesentéricas na década de 1980, há exiguidade de trabalhos na literatura acerca do assunto, indicando a necessidade de estudos randomizados e meta-análises para orientar os rumos no tratamento da isquemia mesentérica.

\section{CONFLITO DE INTERESSES}

Os autores declararam inexistência de conflito de interesses relacionado a este manuscrito.

\section{REFERÊNCIAS BIBLIOGRÁFICAS}

1. Boley SJ, Brandt LJ, Sammartano RJ. History of mesenteric ischemia. The evolution of a diagnosis and management. Surg Clin North Am. 1997;77(2):275-88.

2. Schoots IG, Levi MM, Reekers JA, Lameris JS, van Gulik TM Thrombolytic therapy for acute superior mesenteric artery occlusion. J Vasc Interv Radiol. 2005;16(3):317-29.

3. Herbert GS, Steele SR. Acute and chronic mesenteric ischemia. Surg Clin North Am. 2007;87(5):1115-34.

4. Martinez JP, Hogan GJ. Mesenteric ischemia. Emerg Med Clin North Am. 2004;22(4):909-28.

5. Paterno F, Longo WE. The etiology and pathogenesis of vascular disorders of the intestine. Radiol Clin North Am. 2008;46(5):877-85.

6. Acosta S, Ogren $M$, Sternby $N H$, Bergqvist D, Björck $M$ Incidence of acute thrombo-embolic occlusion of the superior mesenteric artery - a population based study. Eur J Vasc Endovasc Surg. 2004;27(2):145-50.

7. Hackworth CA, Leef JA. Percutaneous transluminal mesenteric angioplasty. Surg Clin North Am. 1997;77(2):371-80.

8. Montgomery RA, Venbrux AC, Bulkley GB. Mesenteric vascular insufficiency. Curr Probl Surg.1997;34(12):943-1025.

9. Nogueira AMMF, Barbosa AJA, Brasileiro Filho G, Raso P. Tubo digestivo. Peritônio. In: Brasileiro Filho G, editor. Bogliolo patologia. Rio de Janeiro: Guanabara Koogan; 2006. p.690-2.

10. Bradbury AW, Brittenden J, McBride K, Ruckley CV. Mesenteric 
ischaemia: a multidisciplinary approach. Br J Surg. 1995;82(11): 1446-59. Comment in: Br J Surg. 1996;83(5):709-10.

11. Brandt LJ. Distúrbios vasculares do intestino. In: Goldman L, Ausiello D, editores. Cecil: tratado de medicina interna. Rio de Janeiro: Elsevier; 2005. p.1009-17.

12. Brandt LJ, Boley SJ. Colonic ischemia. Surg Clin North Am. 1992;72(1):203-29.

13. Gearhart SL. Insuficiência vascular mesentérica. In: Kasper DL, Braunwald E, Fauci AS, Hauser SL, Longo DL, Jameson $J \mathrm{~L}$, editores. Harrison medicina interna. Rio de Janeiro: McGrawHill; 2009. p.1910-12.

14. Kougias P, Lau D, El Sayed H, Zhou W, Huynh TT, Lin PH. Determinants of mortality and treatment outcome following surgical interventions for acute mesenteric ischemia. J Vasc Surg. 2007;46(3):467-74

15. Savassi-Rocha PR, Veloso LF. Insuficiência vascular mesentérica. In: Castro LP, Coelho LGV, editores. Gastroenterologia. Rio de Janeiro: Medsi; 2004. p.1309-24.

16. Stoney R, Cunningham CG. Acute mesenteric ischemia. Surgery. 1993;114(3):489-90.

17. Simi AC. Isquemia intestinal. In: Maffei FHA, editor. Doenças vasculares periféricas. Rio de Janeiro: Medsi; 2002. p.1239-57.

18. Belkin M, Whittemore AD, Donaldson MC, Conte MS, Gravereaux E. Doença oclusiva arterial periférica. In: Townsend CM, editor. Sabiston: tratado de cirurgia. Rio de Janeiro: Elsevier; 2005. p.2022-9.

19. Hirsch AT, Haskal ZJ, Hertzer NR, Bakal CW, Creager MA, Halperin JL, Hiratzka LF, Murphy WR, Olin JW, Puschett JB, Rosenfield KA, Sacks D, Stanley JC, Taylor LM Jr, White C), White J, White RA, Antman EM, Smith SC Jr, Adams CD, Anderson JL, Faxon DP, Fuster V, Gibbons RJ, Hunt SA, Jacobs AK, Nishimura R, Ornato JP, Page RL, Riegel B; American Association for Vascular Surgery; Society for Vascular Surgery; Society for Cardiovascular Angiography and Interventions; Society for Vascular Medicine and Biology; Society of Interventional Radiology; ACC/AHA Task Force on Practice Guidelines Writing Committee to Develop Guidelines for the Management of Patients With Peripheral Arterial Disease; American Association of Cardiovascular and Pulmonary Rehabilitation; National Heart, Lung, and Blood Institute; Society for Vascular Nursing; TransAtlantic InterSociety Consensus; Vascular Disease Foundation. ACC/AHA 2005 Practice Guidelines for the management of patients with peripheral arterial disease (lower extremity, renal, mesenteric, and abdominal aortic): a collaborative report from the American Association for Vascular Surgery/Society for Vascular Surgery, Society for Cardiovascular Angiography and Interventions, Society for Vascular Medicine and Biology, Society of Interventional Radiology, and the ACC/AHA Task Force on Practice
Guidelines (Writing Committee to Develop Guidelines for the Management of Patients With Peripheral Arterial Disease): endorsed by the American Association of Cardiovascular and Pulmonary Rehabilitation; National Heart, Lung, and Blood Institute; Society for Vascular Nursing; TransAtlantic InterSociety Consensus; and Vascular Disease Foundation. Circulation. 2006;113(11):e463-654.

20. Buchardt Hansen HJ. Abdominal angina. Results of arterial reconstruction in 12 patients. Acta Chir Scand. 1976;142(4): 319-25.

21. Brown DJ, Schermerhorn ML, Powell RJ, Fillinger MF, Rzucidlo EM, Walsh DB, et al. Mesenteric stenting for chronic mesenteric ischemia. J Vasc Surg. 2005;42(2):268-74.

22. Zwolak RM, Fillinger MF, Walsh DB, LaBombard FE, Musson A, Darling CE, et al. Mesenteric and celiac duplex scanning: a validation study. J Vasc Surg. 1998;27(6):1078-87; discussion 1088.

23. Hansen KJ, Wilson DB, Craven TE, Pearce JD, English WP, Edwards MS, et al. Mesenteric artery disease in the elderly. J Vasc Surg. 2004;40(1):45-52.

24. Ozden N, Gurses B. Mesenteric ischemia in the elderly. Clin Geriatr Med. 2007;23(4):871-87.

25. Cho JS, Carr JA, Jacobsen G, Shepard AD, Nypaver TJ, Reddy D). Long-term outcome after mesenteric artery reconstruction: a 37-year experience. J Vasc Surg. 2002;35(3):453-60.

26. Allen RC, Martin GH, Rees CR, Rivera FJ, Talkington CM, Garret WV, et al. Mesenteric angioplasty in the treatment of chronic intestinal ischemia. J Vasc Surg. 1996;24(3):415-21; discussion 42-3.

27. Matsumoto AH, Angle JF, Spinosa DJ, Hagspiel KD, Cage $\mathrm{DL}$, Leung DA, et al. Percutaneous transluminal angioplasty and stenting in the treatment of chronic mesenteric ischemia: results and longterm follow up. J Am Coll Surg. 2002;194 (Suppl 1):S22-31.

28. Silva JA, White CJ, Collins TJ, Jenkins JS, Andry ME, Reilly $\mathrm{JP}$, et al. Endovascular therapy for chronic mesenteric ischemia. J Am Coll Cardiol. 2006;47(5):944-50.

29. Kasirajan K, O'Hara PJ, Gray BH, Hertzer NR, Clair DG, Greenberg RK, et al. Chronic mesenteric ischemia: open surgery versus percutaneous angioplasty and stenting. J Vasc Surg. 2001;33(1):63-71

30. Sivamurthy N, Rhodes JM, Lee D, Waldman DL, Green RM, Davies MG. Endovascular versus open mesenteric revascularization: immediate benefits do not equate with short-term functional outcomes. J Am Coll Surg. 2006;202(6):859-67.

31. Zerbib P, Lebuffe G, Sergent-Baudson G, Chamatan A, Massouille D, Lions $C$, et al. Endovascular versus open revascularization for chronic mesenteric ischemia: a comparative study. Langenbecks Arch Surg. 2008;393(6):865-70. 